\title{
Single-particle dynamics in theoretical minimum emittance cell
}

\author{
Yunhai Cai \\ SLAC National Accelerator Laboratory, 2575 Sand Hill Road, Menlo Park, California 94205, USA
}

(Received 27 July 2018; published 19 November 2018)

\begin{abstract}
We have developed a parametrization of the linear optics in a theoretical minimum emittance cell with three quadrupoles. It consists of five independent parameters: two phase advances, a dimensionless horizontal beta function at the center of the dipole, and a bending angle $\phi$ and a length $L$ of the dipole. For the zero chromaticity cell, we again find that the dynamic aperture in the normalized phase space is scaled according to, $\bar{A} \propto \phi \sqrt{L}$. Moreover, we study nonlinear dynamics near the third-order coupled resonances in the framework of the resonance normal form. In particular, we derive the effective Hamiltonians using the Lie algebra method and show that the periodic orbits in tracking can be interpreted as solutions of the Hamilton's equations. Surprisingly, we discover that the scheme is not only applicable to the single resonance but also to the double resonances.
\end{abstract}

DOI: 10.1103/PhysRevAccelBeams.21.114002

\section{INTRODUCTION}

Historically, the theoretical minimum emittance (TME) cell [1] has played an essential role in the advance to a lower emittance in the electron storage rings. Teng illustrated how the equilibrium emittance is scaled according to the beam energy and deflecting angle of the dipole and perhaps, most importantly, how it can be minimized to reach its theoretical minimum without a specific design of the cell. The optimization scheme was further advanced by Potier and Rivkin $[2,3]$ who characterized the cell with a set of the detuned parameters, namely the ratio to the minimum values, relating them to the horizontal phase advance. The parameterization laid a solid foundation for a systematic approach [4] to the design of the low-emittance storage rings [5] and the damping rings [6]. The parametrization provides a strategical guidance for designing a cell, but it still relies on a computer program to find a periodic solution of the cell. In this sense, the optimization scheme is not yet complete.

In this paper, we will develop a complete parametrization of the TEM cell that consists of a dipole magnet, three quadrupoles, and three sextupoles. For simplicity, the sextupoles are set to make the chromaticity zero. The system is chosen to be a good approximation of the TME lattice and yet simple enough to be studied analytically. For single-particle dynamics, we will continue the work of the parameterized alternating focusing and defocusing (FODO) cell [7] and

Published by the American Physical Society under the terms of the Creative Commons Attribution 4.0 International license. Further distribution of this work must maintain attribution to the author(s) and the published article's title, journal citation, and DOI. explore applications of the Kolmogorov-Arnold-Moser (KAM) theory [8-11] to particle accelerators. To avoid repetition, our focus will be placed only on the invariant tori near the nonlinearly coupled resonances. Mostly, the resonance studies [12-14] were carried out in the framework of the canonical perturbation theory. Here, our investigation will be from a modern viewpoint of the Lie algebra [15] with an emphasis on comparisons to direct tracking.

We will introduce a geometrical parametrization of the focusing system based on the paraxial optics and apply it to a half cell with a reflection symmetry in Sec. II. Continuing in Sec. III, we will develop the linear optics, derive the emittance, and then compensate the chromaticities in the periodic cell. Nonlinear aberrations will be given with a brief outline in Sec. IV using the Lie algebra method. Most importantly, we will study the Hamiltonian dynamics in comparison to the tracking in Secs. V and VI for one and two resonances respectively. Finally, in Sec. VII, we will make some concluding remarks. A solution of the cubic equation and the detailed derivation of the effective Hamiltonian [16] in a vicinity of resonance and normal form [17] will be given in the Appendix.

\section{PARAMETRIZATION}

\section{A. Paraxial optics}

The locations of the focusing point relative to its principle plane illustrated in Fig. 1 characterize a conventional optical system. Here, we will apply these well-known properties to the optics of charged particles in the transverse dimensions, namely the horizontal or vertical plane.

Given a 2-by-2 R-matrix [18] of the optical system, we can show, 


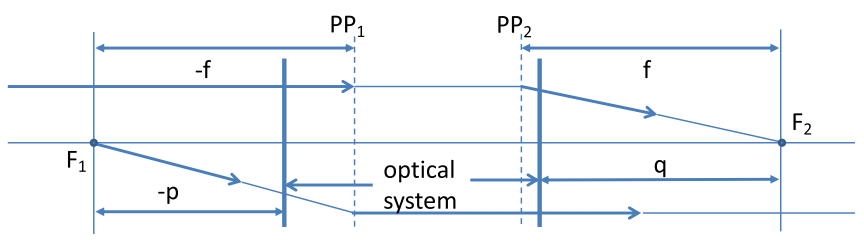

in plane

out plane

FIG. 1. Geometrical parameters in a conventional optical system. $\mathrm{F}_{1,2}$ are the focus points, $\mathrm{PP}_{1,2}$ the principal planes, and $f$ the focus length of the optical system.

$$
p=\frac{M_{22}}{M_{21}}, \quad q=-\frac{M_{11}}{M_{21}}, \quad f=-\frac{1}{M_{21}},
$$

where $M_{11}, M_{21}$, and $M_{22}$ are the elements of the R-matrix. The derivation requires the symplecticity of the R-matrix and validity of the paraxial approximation, $\tan \theta \approx \theta$, where $\theta$ is the angle of the optical ray.

Inversely, given these geometrical parameters in the optical system, we can rewrite its R-matrix as,

$$
M=\left(\begin{array}{cc}
\frac{q}{f} & \frac{1}{f}\left(p q+f^{2}\right) \\
-\frac{1}{f} & -\frac{p}{f}
\end{array}\right) .
$$

\section{B. Doublet}

Let us consider a doublet drawn schematically in Fig. 2. Its R-matrix can be obtained by multiplying the three R-matrices of the elements. Then applying Eq. (1), we have its geometrical parameters,

$$
\begin{gathered}
p=\frac{f_{1}\left(g-f_{2}\right)}{f_{1}-f_{2}+g}, \quad q=\frac{f_{2}\left(g+f_{1}\right)}{f_{1}-f_{2}+g}, \\
f=\frac{f_{1} f_{2}}{f_{1}-f_{2}+g} .
\end{gathered}
$$

Here we have to use the thin lens calculation for the quadrupoles. Sometimes it is useful to have its inverse,

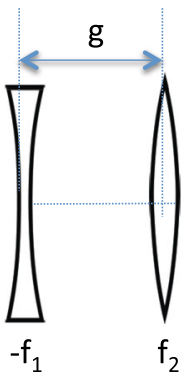

FIG. 2. A schematic layout of a doublet. $f_{1,2}$ are focus lengths of the defocusing and focusing quadrupoles respectively. $g$ is the distance between the quadrupoles.

$$
\begin{gathered}
f_{1}=-\frac{f^{2}+p q}{f-q}, \quad f_{2}=\frac{f^{2}+p q}{f+p}, \\
g=\frac{f^{2}+p q}{f} .
\end{gathered}
$$

To focus the charged particles in both transverse planes simultaneously, a doublet is often required because a magnetic quadrupole focuses in one plane while defocuses in the other.

\section{A half cell}

We now consider a half of a cell with a reflection symmetry as shown in Fig. 3, where the position $s_{1}$ is the starting and $s_{2}$ the reflecting points. Here we represent the doublet with their geometrical parameters, $p, q$, and $f$. And $L_{1}, L_{2}$ are the distances between the doublet to $s_{1}, s_{2}$ respectively.

Using the matrix in Eq. (2) for the doublet and the matrix of drift, we compute the transfer matrix from $s_{1}$ to $s_{2}$ and find,

$$
M_{s_{1} \rightarrow s_{2}}=\left(\begin{array}{cc}
\frac{q-L_{2}}{f} & \frac{1}{f}\left[\left(p+L_{1}\right)\left(q-L_{2}\right)+f^{2}\right] \\
-\frac{1}{f} & -\frac{\left(p+L_{1}\right)}{f}
\end{array}\right) .
$$

Note that the matrix has the same functional form in Eq. (2) with replacements $q \rightarrow q-L_{2}$ and $p \rightarrow p+L_{1}$. This property is consistent with the interpretation of the geometrical parameters, $p, q$, and $f$. Also, the matrix can be represented by [19],

$$
M_{s_{1} \rightarrow s_{2}}=\left(\begin{array}{cc}
\sqrt{\frac{\beta_{2}}{\beta_{1}}} \cos \pi \nu & \sqrt{\beta_{1} \beta_{2}} \sin \pi \nu \\
-\frac{1}{\sqrt{\beta_{1} \beta_{2}}} \sin \pi \nu & \sqrt{\frac{\beta_{1}}{\beta_{2}}} \cos \pi \nu
\end{array}\right),
$$

where $\beta_{1}, \beta_{2}$ are the beta functions [20] at the positions $s_{1}, s_{2}$ respectively, $\nu$ the betatron tune of the cell. Here we have used the property of the reflection points, namely $\alpha_{1}=\alpha_{2}=0$. Comparing it with the matrix in Eq. (5), we obtain,

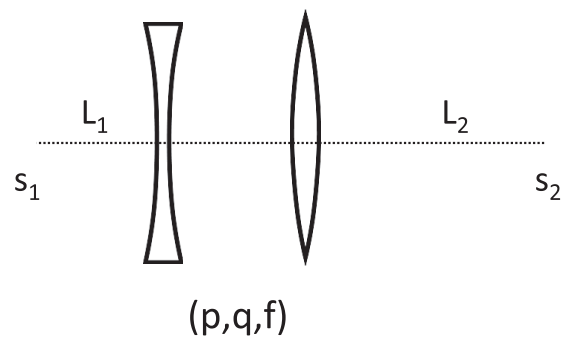

FIG. 3. A schematic layout of a half cell for a cell with a reflection symmetry. $p, q$, and $f$ are the geometrical parameters of the doublet. $L_{1}, L_{2}$ are the distances to the entry and exit of the half cell respectively. 


$$
\begin{aligned}
& p=-L_{1}-\beta_{1} \cot \pi \nu, \\
& q=L_{2}+\beta_{2} \cot \pi \nu, \\
& f=\sqrt{\beta_{1} \beta_{2}} \csc \pi \nu .
\end{aligned}
$$

Given these geometrical parameters, the focus lengths of the quadrupoles and separation distance can be calculated. Substituting Eq. (7) into Eq. (4), we have,

$$
\begin{aligned}
f_{1} & =\frac{\beta_{1} \beta_{2}-L_{1} L_{2}-\left(L_{2} \beta_{1}+L_{1} \beta_{2}\right) \cot \pi \nu}{L_{2}+\beta_{2} \cot \pi \nu-\sqrt{\beta_{1} \beta_{2}} \csc \pi \nu}, \\
f_{2} & =\frac{L_{1} L_{2}-\beta_{1} \beta_{2}+\left(L_{2} \beta_{1}+L_{1} \beta_{2}\right) \cot \pi \nu}{L_{1}+\beta_{1} \cot \pi \nu-\sqrt{\beta_{1} \beta_{2}} \csc \pi \nu}, \\
g & =\frac{\left(\beta_{1} \beta_{2}-L_{1} L_{2}\right) \sin \pi \nu-\left(L_{2} \beta_{1}+L_{1} \beta_{2}\right) \cos \pi \nu}{\sqrt{\beta_{1} \beta_{2}}} .
\end{aligned}
$$

The formulas in this section are equally applicable to either the horizontal or vertical plane. In this paper, we choose the horizontal lattice functions to fix the physical parameters such as the quadrupole strengths since one of our main concerns is the natural emittance, to which the horizontal tune: $\nu$ and the beta function: $\beta_{2}$ at the center of the bending dipole play essential roles $[2,3]$.

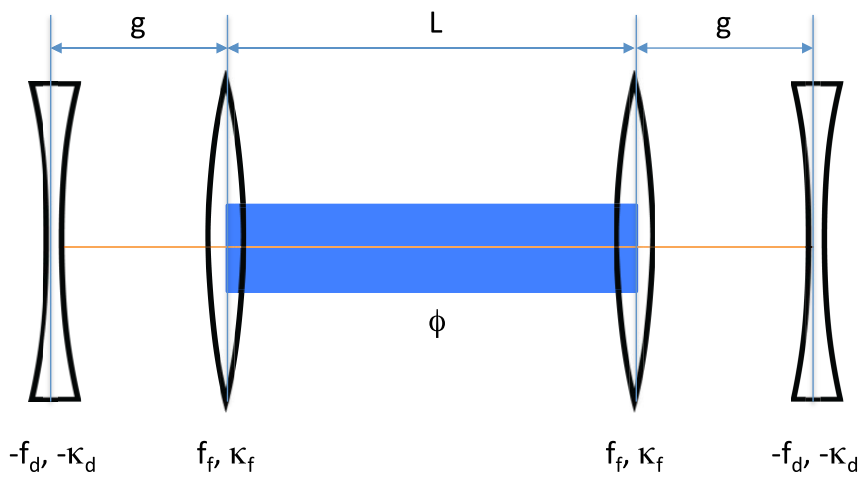

FIG. 4. A periodic theoretical minimum emittance cell with dipole, quadrupole, and sextupole magnets. $\phi$ and $L$ are the bending angle and length of the dipole, $f_{f, d}$ the focus lengths of the quadrupoles, $\kappa_{f, d}$ the strengths of the sextupoles.

\section{PERIODIC CELL}

\section{A. Layout}

We would like to illustrate how the map works using a periodic TME cell that contains three quadrupoles as shown in Fig. 4. The cell is chosen because it contains the most essential ingredients in the common TME cell and yet is analytically solvable. The quadrupoles and sextupoles are lumped together as a thin multipole with a sector bending dipole in between. Here $f_{f}$ and $f_{d}$ are the focal lengths of the focusing and de-focusing quadrupoles, respectively. Also $\phi$ and $L$ are the bending angle and length of the dipole.

Applying Eq. (8) with a substitution of $L_{1}=0, L_{2}=$ $L / 2, f_{1}=2 f_{d}$ and $f_{2}=f_{f}$, we find the settings of the doublet,

$$
\begin{aligned}
& \bar{f}=\frac{\sqrt{\bar{\beta}_{1}}\left(\cot \pi \nu-2 \bar{\beta}_{2}\right)}{2\left(\sqrt{\bar{\beta}_{1}} \cot \pi \nu-\sqrt{\bar{\beta}_{2}} \csc \pi \nu\right)}, \\
& \bar{d}=\frac{\bar{\beta}_{1}\left(2 \bar{\beta}_{2}-\cot \pi \nu\right)}{2\left(1+2 \bar{\beta}_{2} \cot \pi \nu-2 \sqrt{\bar{\beta}_{1} \bar{\beta}_{2}} \csc \pi \nu\right)}, \\
& \bar{g}=\frac{\sqrt{\bar{\beta}_{1}}\left(2 \bar{\beta}_{2} \sin \pi \nu-\cos \pi \nu\right)}{2 \sqrt{\bar{\beta}_{2}}},
\end{aligned}
$$

where $\bar{d}=f_{d} / L, \bar{f}=f_{f} / L$ are the dimensionless focusing lengths of the quadrupoles, normalized by the bend length $L$. Similarly, we use "bar" to note the scaling of $L$ for the other parameters in the formulas. As we will show later, $\bar{\beta}_{1,2}$ can also be represented by a function of the betatron tunes. As a result, these dimensionless parameters can be plotted as a function of the betatron tunes as shown in Fig. 5. The figure shows that higher horizontal tune or smaller emittance is essentially achieved by a combination of stronger focusing quadrupole and larger separation between the quadrupoles. The larger the spacing between the quadrupoles in the doublet, the smaller the packing factor of the magnets is. This is a significant drawback in the TME cell at high tunes.
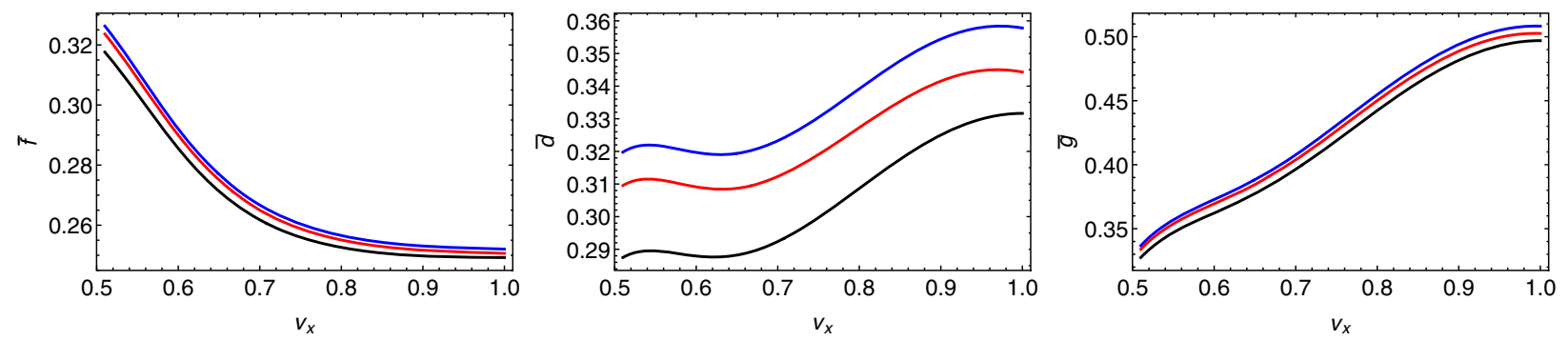

FIG. 5. Dimensionless parameters of the doublet as a function of the betatron tunes with various ratios: $\nu_{y}: \nu_{x}=1: 4,1: 3$, and $1: 2$ represented by blue, red, and black color respectively. 


\section{B. Optics}

Given the importance of the dipole in generating the natural emittance, we choose the reference point in the middle of the dipole. The transfer map $\mathcal{M}_{\text {cell }}$ of the cell can be obtained by initializing an identity map and then concatenating it through the maps of the elements. Here we use the explicit maps of Eqs. (2.5) and (2.6) in
Ref. [21] for the bends and kicks respectively. The computation is carried out using Mathematica [22]. Taking the Jacobian of the transfer map for the R-matrix and then comparing it with the Courant-Synder matrix [20] of a periodical system, we find that the betatron tunes, defined as the phase advances in units of $2 \pi$, are given by,

$$
\begin{aligned}
& \nu_{x}=1-\frac{1}{2 \pi} \cos ^{-1}\left\{\frac{2 \bar{d}\left[\bar{f}^{2}+\bar{g}-\bar{f}(2 \bar{g}+1)\right]+(\bar{f}-\bar{g})[\bar{f}(2 \bar{g}+1)-\bar{g}]}{2 \bar{d} \bar{f}^{2}}\right\}, \\
& \nu_{y}=\frac{1}{2 \pi} \cos ^{-1}\left\{\frac{2 \bar{d}\left[\bar{f}^{2}+\bar{g}+\bar{f}(2 \bar{g}+1)\right]-(\bar{f}+\bar{g})[\bar{f}(2 \bar{g}+1)+\bar{g}]}{2 \bar{d} \bar{f}^{2}}\right\} .
\end{aligned}
$$

Moreover, we have the beta functions at the center of the dipole,

$$
\begin{aligned}
& \beta_{x}=\frac{[4 \bar{d} \bar{f}-2 \bar{d}+\bar{f}(2 \bar{g}+1)-\bar{g}][\bar{f}(2 \bar{g}+1)-\bar{g}] L}{4 \bar{d} \bar{f}^{2} \sin 2 \pi \nu_{x}}, \\
& \beta_{y}=\frac{[4 \bar{d} \bar{f}+2 \bar{d}-\bar{f}(2 \bar{g}+1)-\bar{g}][\bar{f}(2 \bar{g}+1)+\bar{g}] L}{4 \bar{d} \bar{f}^{2} \sin 2 \pi \nu_{y}},
\end{aligned}
$$

and the horizontal dispersion,

$$
\eta_{x}=\frac{(8 \bar{d} \bar{f}+4 \bar{f} \bar{g}-2 \bar{d}+\bar{f}-\bar{g}) L \phi}{8(2 \bar{d}-\bar{f}+\bar{g})} .
$$

$\alpha_{x, y}=0$ and $\eta_{p_{x}}=0$ due to the reflection symmetry.

\section{Emittance}

Substituting the physical parameters in Eq. (9) into Eqs. (10)-(12) for the optical functions in the horizontal plane, we obtain,

$$
\nu_{x}=\nu, \quad \beta_{x}=\bar{\beta}_{2} L, \quad \eta_{x}=\frac{1}{8} L \phi\left(1+4 \bar{\beta}_{2} \cot \pi \nu\right) .
$$

The first two equations are merely a consistency check of the parametrization introduced in the previous section. The third equation, namely the relationship between the horizontal tune and dispersion, was first derived by Rivkin [3]. Given the beta and dispersion functions, we evaluate the radiation integrals [23] and derive the form factor,

$$
F=\frac{1+10 \bar{\beta}_{2}^{2}+10 \bar{\beta}_{2} \cot \pi \nu+30 \bar{\beta}_{2}^{2} \cot ^{2} \pi \nu}{120 \bar{\beta}_{2}},
$$

which is defined by the natural emittance $\epsilon_{x}=C_{q} F \gamma^{2} \phi^{3}$ where $C_{q}=3.8319 \times 10^{-13} \mathrm{~m}$ and $\gamma$ is the Lorentz factor. It can be minimized and reduced to,

$$
F=\frac{1}{60}\left(5 \cot \pi \nu+\sqrt{10+30 \cot ^{2} \pi \nu}\right),
$$

by setting,

$$
\bar{\beta}_{2}=\frac{1}{\sqrt{10+30 \cot ^{2} \pi \nu}}
$$

To minimize the emittance, we always use this optimal value of $\bar{\beta}_{2}$ in this paper.

As shown in Fig. 6, it can be further minimized by selecting: $\nu_{\min }=1-\frac{1}{\pi} \cot ^{-1} \sqrt{5 / 3}$ with the well-known minimum: [1] $F_{\min }=1 / 12 \sqrt{15}$. The reduction factor of the emittance from $\nu=0.5$ to $\nu_{\min }$ is about 2.45 .

\section{Linear stability}

Similarly, we can calculate the optical parameters in the vertical plane. In particular, we find that the betatron tune is given by,

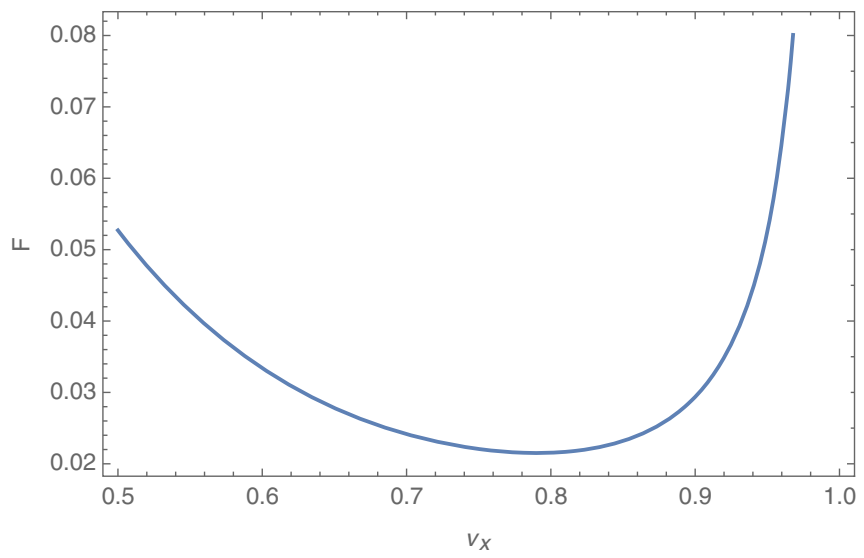

FIG. 6. The form factor of emittance in Eq. (15) as a function of the horizontal betatron tune. 

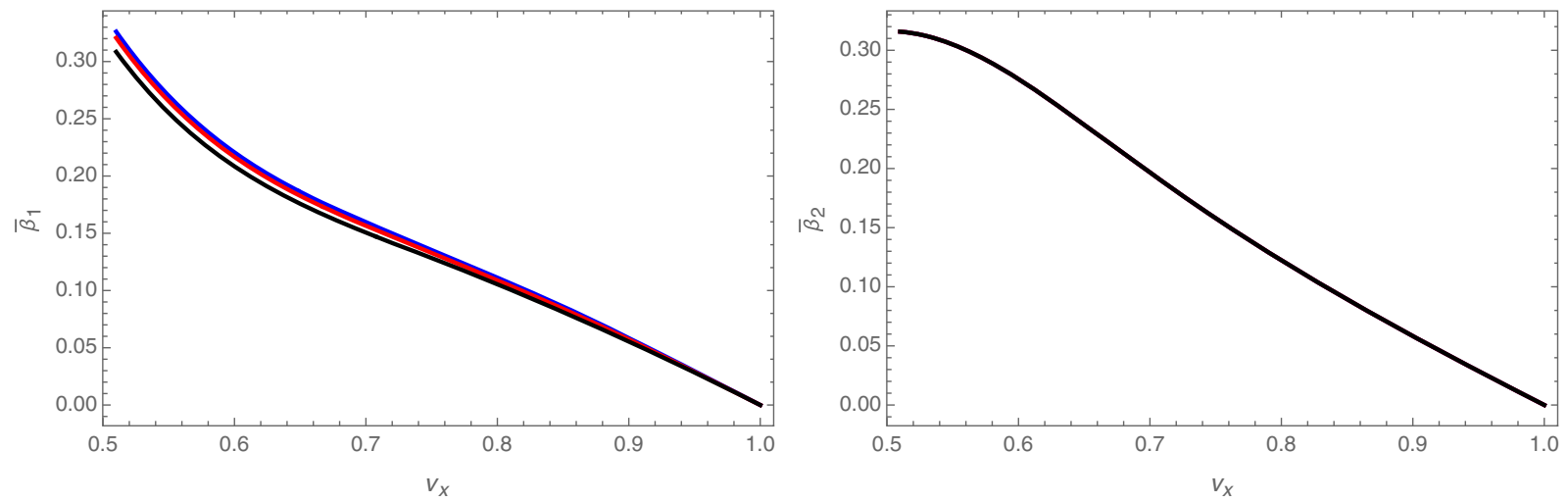

FIG. 7. The horizontal beta functions at the end (left) and center (right) of the cell as a function of the betatron tunes with various ratios: $\nu_{y}: \nu_{x}=1: 4,1: 3$, and $1: 2$ represented by blue, red, and black color respectively.

$$
\begin{aligned}
\cos 2 \pi \nu y= & \frac{1}{2 \bar{\beta}_{1} \bar{\beta}_{2}\left(2 \bar{\beta}_{2} \sin \pi \nu-\cos \pi \nu\right)}\left\{8 \sqrt{\bar{\beta}_{1} \bar{\beta}_{2}}\left(\bar{\beta}_{1}+4 \bar{\beta}_{2}\right)-\bar{\beta}_{2}\left(47 \bar{\beta}_{1}+16 \bar{\beta}_{2}\right) \cos \pi \nu\right. \\
& +8 \sqrt{\bar{\beta}_{1} \bar{\beta}_{2}}\left(\bar{\beta}_{1}+2 \bar{\beta}_{2}\right) \cos 2 \pi \nu-3 \bar{\beta}_{1} \bar{\beta}_{2} \cos 3 \pi \nu+\left[30 \bar{\beta}_{1} \bar{\beta}_{2}^{2}-\left(\bar{\beta}_{1}+8 \bar{\beta}_{2}\right)\right] \sin \pi \nu \\
& \left.+2 \sqrt{\bar{\beta}_{1} \bar{\beta}_{2}}\left[3-4\left(\bar{\beta}_{1} \bar{\beta}_{2}+\bar{\beta}_{2}^{2}\right)\right] \sin 2 \pi \nu+\bar{\beta}_{1}\left(2 \bar{\beta}_{2}^{2}-1\right) \sin 3 \pi \nu\right\} .
\end{aligned}
$$

This condition has to be satisfied for a stable cell. Essentially, it defines $\bar{\beta}_{1}$ since $\bar{\beta}_{2}$ should be set according to Eq. (16) for a minimal emittance. In fact, $\bar{\beta}_{1}$ as a function of $\nu_{x}, \nu_{y}, \bar{\beta}_{2}$ can be obtained explicitly by solving a cubic equation. The solution is given in Appendix A. As a result, we have found that five independent parameters, namely $\nu_{x}$, $\nu_{y}, \bar{\beta}_{2}, \phi, L$, can characterize a stable TME cell. It is worth noting that the only dimensional parameter is the length of the dipole $L$. The dimensionless horizontal beta functions $\bar{\beta}_{1}$ and $\bar{\beta}_{2}$ as a function of the betatron tunes are shown in Fig. 7. They depend similarly on the horizontal tune and hardly any on the vertical tune.

We check the parameterization for the TME cell at the minimum emittance against the computer program
MAD [24]. In particular, the lattice functions computed numerically using MAD, shown in Fig. 8, excellently agree to the analytical calculations. A drawback of the cell is that the vertical beta function at the defocusing quadrupole is too large. As we will see later, that leads to larger nonlinear aberrations in the vertical plane.

\section{E. Chromatic compensation}

The Courant-Synder parameters with $\delta$ dependence can be calculated [21] using the symplectic maps. In particular, by computing the phase advances up to the first-order of $\delta$, we derive the natural chromaticity,

$$
\begin{aligned}
\xi_{x 0} & =-\frac{\left[4 \bar{d} \bar{f}+3 \bar{g}^{2}-4 \bar{f} \bar{g}(\bar{g}+1)-2 \bar{d} \bar{f}(2 \bar{g}+1)+\bar{f}^{2}(2 \bar{g}+1)\right.}{2 \pi \sqrt{(\bar{f}-\bar{g})(2 \bar{d}-\bar{f}+\bar{g})[\bar{f}(2 \bar{g}+1)-\bar{g}+4 \bar{d} \bar{f}-2 \bar{d}][\bar{f}(2 \bar{g}+1)-\bar{g}]}}, \\
\xi_{y 0} & =-\frac{\left[-4 \bar{d} \bar{f}+3 \bar{g}^{2}+4 \bar{f} \bar{g}(\bar{g}+1)-2 \bar{d} \bar{f}(2 \bar{g}+1)+\bar{f}^{2}(2 \bar{g}+1)\right.}{2 \pi \sqrt{(\bar{f}+\bar{g})(2 \bar{d}-\bar{f}-\bar{g})[\bar{f}(2 \bar{g}+1)+\bar{g}-4 \bar{d} \bar{f}-2 \bar{d}][\bar{f}(2 \bar{g}+1)+\bar{g}]}} .
\end{aligned}
$$

They are plotted in Fig. 9 as a function of the betatron tunes. The amplitude of horizontal chromaticity increases rapidly beyond $\nu_{\min } \approx 0.79$. From the viewpoint of the chromaticity in the vertical plane, the ratio of $\nu_{y}: \nu_{x}=1: 4$ or $1: 3$ seems reasonable while $1: 2$ is too high, largely due to the high vertical beta function at the defocusing quadrupole.
Clearly, we can use the two sextupoles to zero out the natural chromaticity. Solving two linear equations, we find the necessary strengths,

$$
\kappa_{f}=\frac{2(2 \bar{d}-\bar{f}+\bar{g})}{(2 \bar{d}+\bar{g}) \bar{f}^{2} L^{2} \phi}, \quad \kappa_{d}=\frac{(2 \bar{d}-\bar{f}-\bar{g})}{\bar{d}^{2} \bar{f} L^{2} \phi}
$$




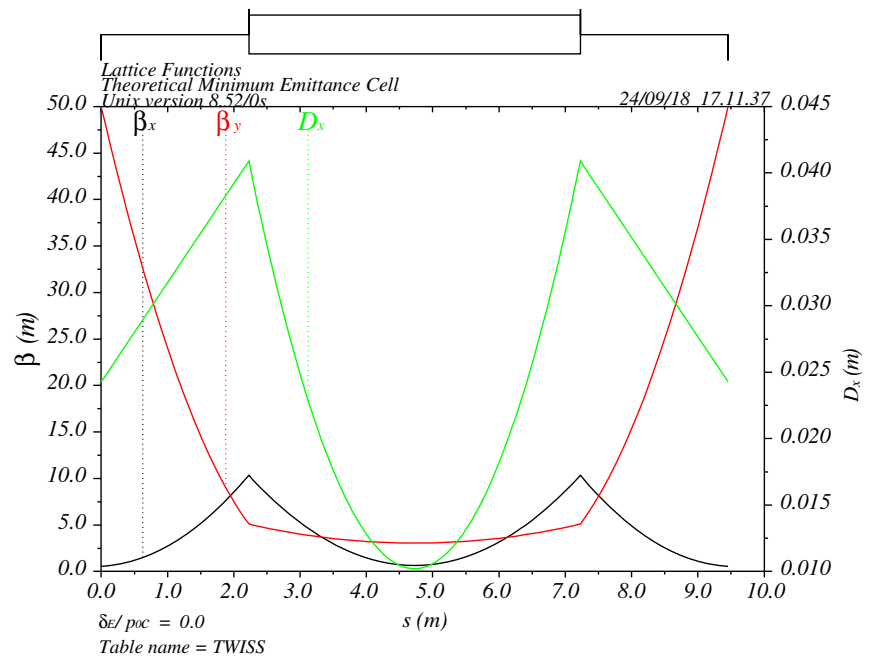

FIG. 8. Lattice functions of TME cell at the minimum emittance with $\nu_{x}=\nu_{\min }, \nu_{y}=\nu_{\min } / 3, \phi=\pi / 64$, and $L=5 \mathrm{~m}$.

It is worth noting that the settings are identical for the values necessary for the local compensation. As a result, the chromaticities are well corrected. With the formulas, we plot the strengths of the sextupoles in Fig. 10, which shows

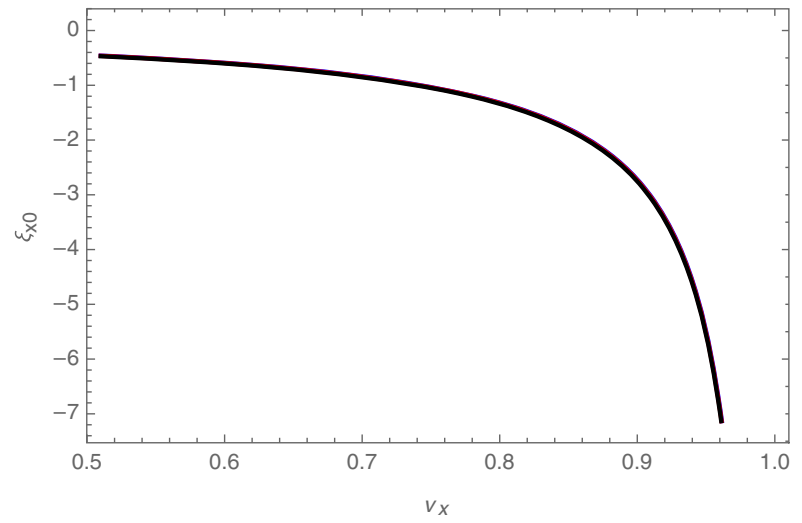

that the defocusing sextupole is stronger and it can be significantly reduced by lowering the vertical tune.

\section{NONLINEARITY}

For simplicity, we set the values of the sextupoles at the zero chromaticity in the study of nonlinear dynamics. It is a good approximation in a single cell because typical circular accelerators contain many cells and each cell shares only little positive chromaticities. Moreover, the zero chromaticity reduces the impact of the path lengthening.

Given the settings of the sextupoles in Eq. (19), the thirdorder Lie polynomial can be derived similarly to the parametrized FODO cell [7] using the Dragt-Finn factorization [25]. Since the chromaticity is well compensated, the chromatic aberration is negligible. The geometric part consists of five resonance driving terms and is given by,

$$
\begin{aligned}
f_{3}= & \frac{1}{\phi \sqrt{L}}\left\{\left(C_{2100} J_{x}^{3 / 2}+C_{1011} J_{x}^{1 / 2} J_{y}\right) \cos \left(\psi_{x}-\pi \nu_{x}\right)\right. \\
& +C_{3000} J_{x}^{3 / 2} \cos 3\left(\psi_{x}-\pi \nu_{x}\right) \\
& +J_{x}^{1 / 2} J_{y}\left[C_{1020} \cos \left(\psi_{x}+2 \psi_{y}-\pi \nu_{x}-2 \pi \nu_{y}\right)\right. \\
& \left.\left.+C_{1002} \cos \left(\psi_{x}-2 \psi_{y}-\pi \nu_{x}+2 \pi \nu_{y}\right)\right]\right\},
\end{aligned}
$$

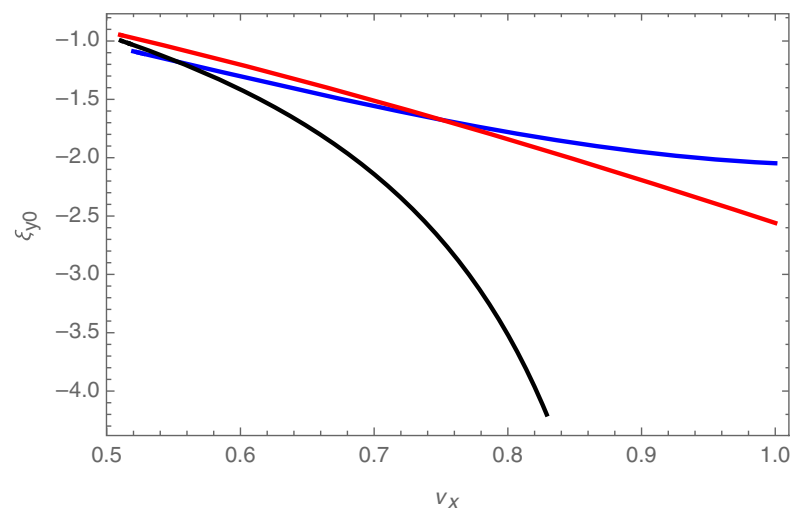

FIG. 9. The natural chromaticity in the horizontal (left) and vertical (right) planes as a function of the betatron tunes with various ratios: $\nu_{y}: \nu_{x}=1: 4,1: 3$, and $1: 2$ represented by blue, red, and black color respectively.
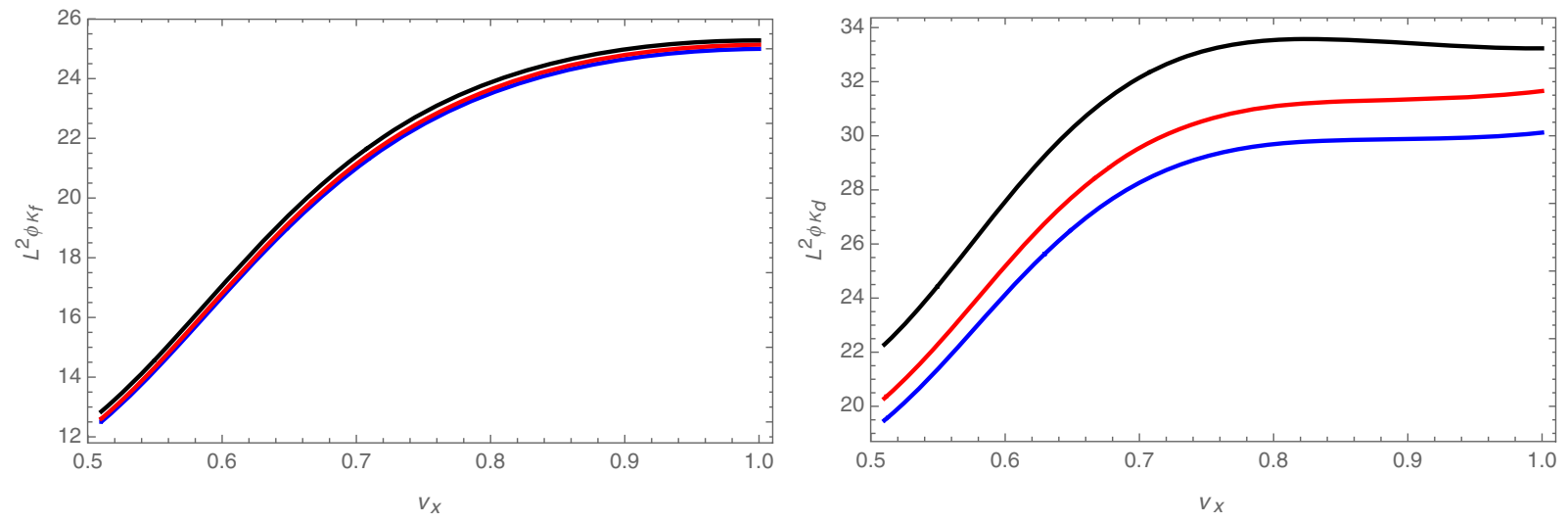

FIG. 10. The settings of focusing (left) and de-focusing (right) sextupoles as a function of the betatron tunes with various ratios: $\nu_{y}: \nu_{x}=1: 4,1: 3$, and $1: 2$ represented by blue, red, and black color respectively. 

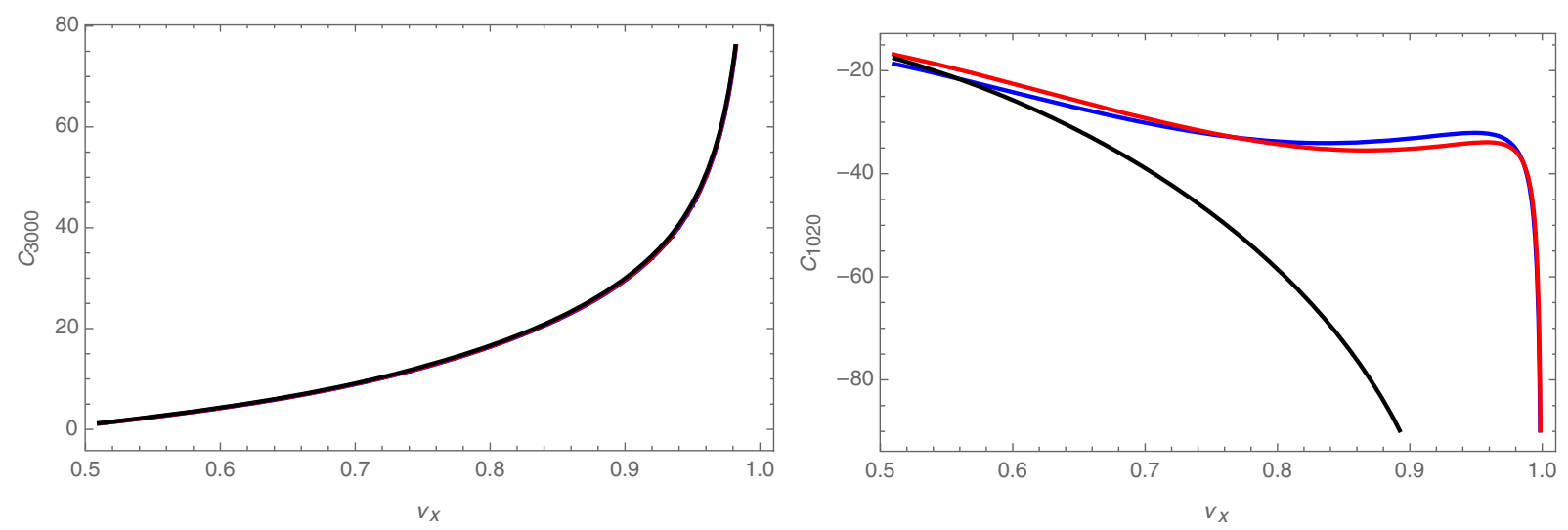

FIG. 11. The coefficients of resonance driving terms: $3 \nu_{x}$ (left) and $\nu_{x}+2 \nu_{y}$ (right) as a function of the betatron tunes with various ratios: $\nu_{y}: \nu_{x}=1: 4,1: 3$, and $1: 2$ represented by blue, red, and black color respectively.

where $J_{x, y}, \psi_{x, y}$ are the action and angle variables [26] in the horizontal and vertical planes respectively. It should be emphasized that the only dependence on the bending angle $\phi$ and length $L$ of the dipole is in a combination of $\phi \sqrt{L}$ in its denominator. This property leads to the scaling law of the dynamic aperture in the normalized coordinates. Here the coefficients $C_{j k l m}$ are functions of the remaining parameters: $\nu_{x, y}$ and $\bar{\beta}_{2}$. Their subscripts indicate the indices of power series in the complex variables.

For the horizontal resonances, their coefficients can be written as,

$$
\begin{aligned}
C_{2100}= & \frac{1}{\mathcal{F}}\left[\sqrt{\bar{\beta}_{1} \bar{\beta}_{2}}\left(1+8 \bar{\beta}_{2}^{2}\right) \cos \pi \nu_{x}-\bar{\beta}_{2}\left(1+4 \bar{\beta}_{1} \bar{\beta}_{2}+4 \bar{\beta}_{2}^{2}-2 \sqrt{\bar{\beta}_{1} \bar{\beta}_{2}} \sin \pi \nu_{x}\right)\right], \\
C_{3000}= & \frac{1}{3 \mathcal{F}}\left[\bar{\beta}_{2}\left(1-4 \bar{\beta}_{1} \bar{\beta}_{2}+4 \bar{\beta}_{2}^{2}\right)+2 \sqrt{\bar{\beta}_{1} \bar{\beta}_{2}}\left(2 \bar{\beta}_{2}^{2}-1\right) \cos \pi \nu_{x}\right. \\
& +2 \bar{\beta}_{2}\left(1-4 \bar{\beta}_{2}^{2}\right) \cos 2 \pi \nu_{x}-\sqrt{\bar{\beta}_{1} \bar{\beta}_{2}}\left(1-4 \bar{\beta}_{2}^{2}\right) \cos 3 \pi \nu_{x} \\
& \left.+6 \bar{\beta}_{2} \sqrt{\bar{\beta}_{1} \bar{\beta}_{2}} \sin \pi \nu_{x}-8 \bar{\beta}_{2}^{2} \sin 2 \pi \nu_{x}+4 \bar{\beta}_{2} \sqrt{\bar{\beta}_{1} \bar{\beta}_{2}} \sin 3 \pi \nu_{x}\right]
\end{aligned}
$$

where $\mathcal{F}=\bar{\beta}_{2}^{2} \sqrt{2 \bar{\beta}_{1}}\left(2 \bar{\beta}_{2}-\cot \pi \nu_{x}\right)$.

Moreover, we obtain the expressions for the three coupled resonances as well. But the formulas are too lengthy to write out. Here we choose to plot the coefficients of two resonances: $3 \nu_{x}$ and $\nu_{x}+2 \nu_{y}$ in Fig. 11. Their amplitudes are largely following the pattern of the natural chromaticity in Fig. 9. This finding agrees with an estimate by Levichev and Kvardakov [27].

To see the effects of the resonances, we use the formulas in Eq. (9) to construct a cell with specified betatron tunes and dipole parameters $\phi=\pi / 64$ and $L=5 \mathrm{~m}$. Setting the sextupoles according to Eq. (19), we scan the dynamic aperture by tracking at various betatron tunes. The averaged dynamic aperture in the normalized coordinates divided by $\phi \sqrt{L}$ is color coded on the map in Fig. 12. The sum resonances: $3 \nu_{x}$ and $\nu_{x}+2 \nu_{y}$ are clearly seen and dominant in the tune scan. The dynamic aperture is more than an order of magnitudes smaller than the one in the parametrized FODO cell [7].

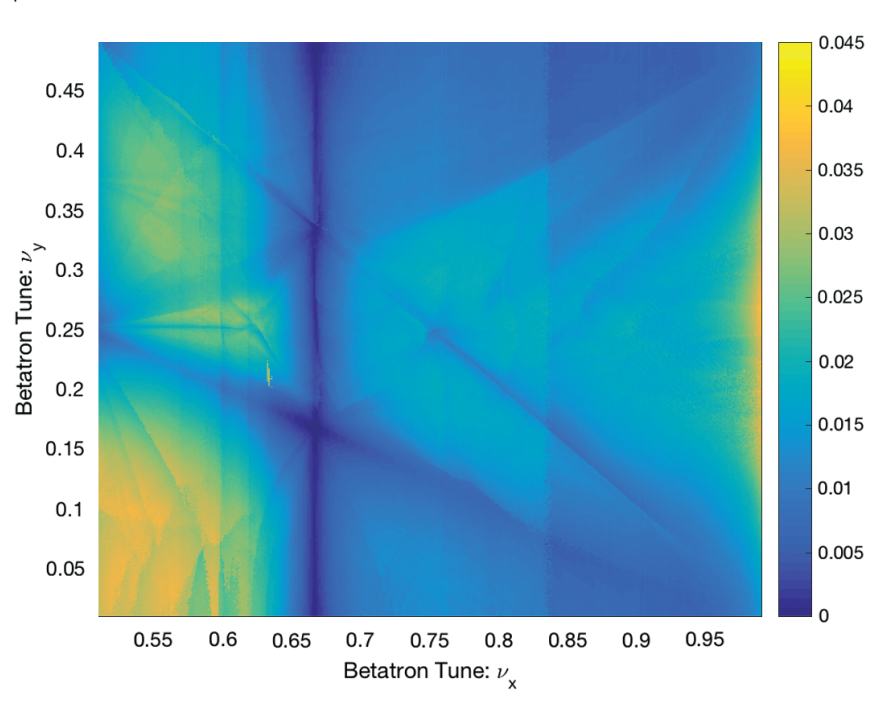

FIG. 12. A tune scan of the dynamic apertures. The color scale represents the averaged dynamic aperture in the normalized coordinates divided by $\phi \sqrt{L}$. 
Taking an example of the TME cell with the minimum emittance shown in Fig. 8, 128 cells make an electron storage ring with a circumference of 1210 meter. At an energy of $6 \mathrm{GeV}$, the natural emittance is $135 \mathrm{pm}$, which reaches the range of the incoming synchrotron light sources [28]. Since there are no dispersion-free straights to place the undulators in the ring, the comparison should be taken only as an estimate. Moreover, the dynamic aperture at the center of the dipole is $1 \mathrm{~mm}$ in the horizontal plane and $2 \mathrm{~mm}$ in vertical one, which is too small for off-axis injection. To increase the dynamic aperture, the phase advances should be carefully selected for cancellation of the resonance driving terms $[4,29]$.

\section{SINGLE RESONANCE}

Near the horizontal resonance $3 \nu_{x}$, we obtain similar results as in the study of the parameterized FODO cell [7]. To avoid repetition, we choose not to write out the finding.

\section{A. Sum resonance}

In the vicinity of the sum resonance: $\nu_{x}+2 \nu_{y}=p+\Delta \nu$, where $p$ is an integer, the effective Hamiltonian can be written as,

$$
\begin{aligned}
\mathcal{H}= & \frac{2 \pi \Delta \nu}{5}\left(J_{x}+2 J_{y}\right) \\
& -\frac{\pi \Delta \nu C_{1020}}{\phi \sqrt{L} \sin \pi(p+\Delta \nu)} J_{x}^{1 / 2} J_{y} \cos \left(\psi_{x}+2 \psi_{y}\right) .
\end{aligned}
$$

A derivation will be given in Appendix B. Rewriting the Hamiltonian in terms of the normalized coordinates, we have,

$$
\begin{aligned}
\mathcal{H}= & \frac{\pi \Delta \nu}{5}\left[\left(\bar{x}^{2}+\bar{p}_{x}^{2}\right)+2\left(\bar{y}^{2}+\bar{p}_{y}{ }^{2}\right)\right] \\
& +\theta\left[\bar{x}\left(\bar{y}^{2}-\bar{p}_{y}{ }^{2}\right)-2 \bar{y} \bar{p}_{x} \bar{p}_{y}\right],
\end{aligned}
$$

where $\theta$ is given by,

$$
\theta=-\frac{\pi \Delta \nu C_{1020}}{2 \phi \sqrt{2 L} \sin \pi(p+\Delta \nu)} .
$$

It is important to know that $K=\left(\bar{x}^{2}+\bar{p}_{x}{ }^{2}\right)-\left(\bar{y}^{2}+\bar{p}_{y}{ }^{2}\right) / 2$ is an invariance in this Hamiltonian system. The invariance can be checked easily by showing that the Poisson bracket of $\mathcal{H}$ and $K$ is zero.

\section{Invariant tori}

Even with the invariance, the general solution of the Hamilton's equation is not known. Here, we would like to find a specific solution,

$$
\begin{aligned}
& \bar{x}=A_{x} \cos (-2 \mu n), \quad \bar{p}_{x}=-A_{x} \sin (-2 \mu n), \\
& \bar{y}=A_{y} \cos (\mu n), \quad \bar{p}_{y}=-A_{y} \sin (\mu n),
\end{aligned}
$$

where $n$ is the turn number as the time variable. Substituting it into the Hamilton's equation, we find that it is indeed a solution provided,

$$
\begin{aligned}
4 \pi \Delta \nu+10 A_{x} \theta-5 \mu & =0, \\
5 A_{y}^{2} \theta+2 A_{x}(\pi \Delta \nu+5 \mu) & =0 .
\end{aligned}
$$

Together with the constant of motion, $K=A_{x}^{2}-A_{y}^{2} / 2$, we solve these equations and obtain,

$A_{x}=\frac{-\pi \Delta \nu+\sqrt{\pi^{2} \Delta \nu^{2}+12 K \theta^{2}}}{6 \theta}$,

$A_{y}=\frac{1}{3} \sqrt{\frac{\pi^{2} \Delta \nu^{2}-12 K \theta^{2}-\pi \Delta \nu \sqrt{\pi^{2} \Delta \nu^{2}+12 K \theta^{2}}}{\theta^{2}}}$.

Note that there are other solutions but they are not seen in the tracking.

The solution requires that the condition, $\pi^{2} \Delta \nu^{2}+$ $12 K \theta^{2} \geq 0$, is satisfied. Most importantly, the largest invariant tori is determined by, $\pi^{2} \Delta \nu^{2}+12 K \theta^{2}=0$, and we have,

$$
A_{x}^{(\max )}=-\frac{\pi \Delta \nu}{6 \theta}, \quad A_{y}^{(\max )}=\frac{\sqrt{2}}{3}\left|\frac{\pi \Delta \nu}{\theta}\right| .
$$

The special solution is compared against tracking with the same initial condition in the vertical plane as shown in Fig. 13. The large amplitudes, the bigger deviation is between the tracking and analytical solution, indicating strongly the higher order nonlinear effects in the tracking. The equal increment beyond the largest tori shown in the figure is unstable in tracking. This is predicted according to the maximal tori in Eq. (28), which is plotted as the black lines in the figure.

It is worth noting that these invariant tori were first discovered in the Hamiltonian perturbation theory by Franchetti and Schmidt [30], who called them "fixed lines" in the 4D phase space. In fact, they are the periodic orbits.

\section{Dynamic aperture}

In the design of storage rings, an adequate dynamic aperture is often required. To compute the dynamic aperture, we track the particles with different amplitudes as shown in Fig. 14.

Taking a similar approach for the parametrized FODO cell [7], we would like to examine contours defined by a constant value of the Hamiltonian in Eq. (23) and find a singular contour. The difference here is that there is another constant of motion $K$. This allows us first to solve $\bar{p}_{x}$ with a fixed value of $K$ and substitute it into the Hamiltonian and then solve $\bar{p}_{y}$. We find a singularity at, 

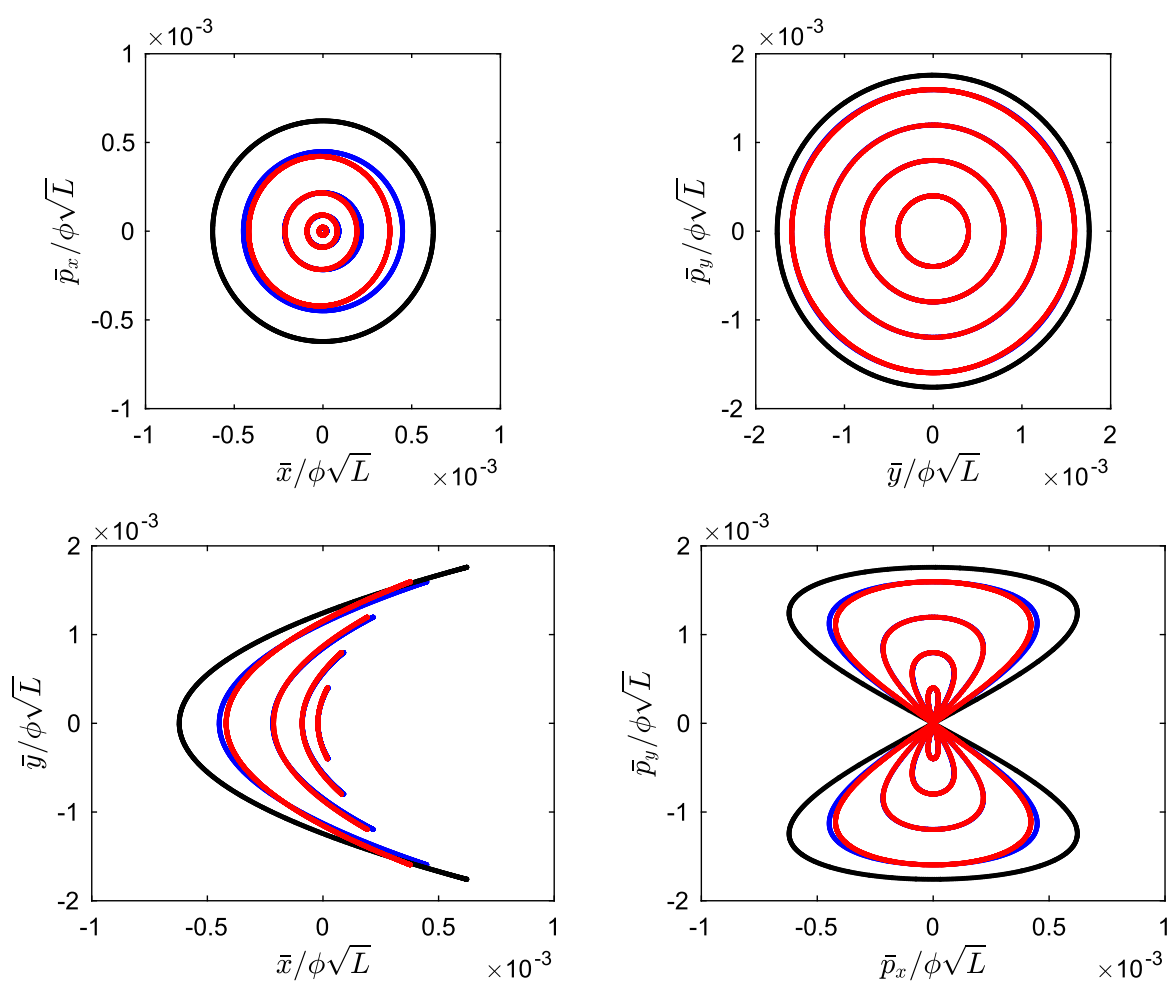

FIG. 13. A set of invariant tori in the normalized 4D phase spaces near the sum resonance: $\nu_{x}+2 \nu_{y}$ with $\nu_{x}=0.618$ and $\nu_{y}=0.196$. The red dots represent the periodic orbits from tracking with bend length $L=5 \mathrm{~m}$ and angle $\phi=\pi / 64$, the blue lines are the special solutions of the Hamiltonian in Eq. (23) with $\Delta \nu=0.01$ and $\theta=-76.73 \mathrm{~m}^{-1 / 2}$, the black lines are the maximal tori predicted by the theory.

$$
\bar{y}=\frac{1}{\sqrt{2}} \bar{x}-\frac{\pi \Delta \nu}{2 \sqrt{2} \theta} .
$$

This singularity defines a contour that leads to infinity in the direction of the momentum $\bar{p}_{y}$. This line along with an arc with a radius, $\sqrt{\left(A_{x}^{(\max )}\right)^{2}+\left(A_{y}^{(\max )}\right)^{2}}=\left|\frac{\pi \Delta \nu}{2 \theta}\right|$, are plotted in Fig. 14 for a comparison to the tracking. The theory gives a reasonably good estimate of the dynamic aperture.

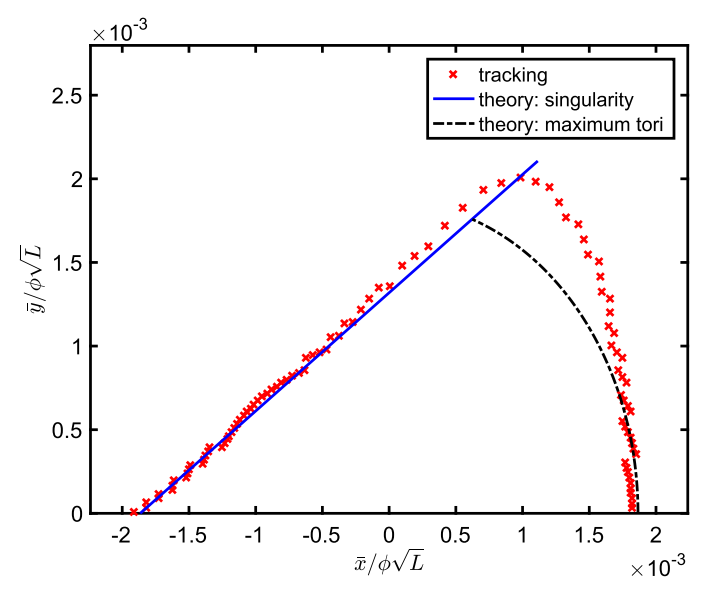

FIG. 14. Comparison of the dynamic apertures between the theory (blue solid and black dashed lines) and the tracking (red cross) at a vicinity of the sum resonance: $\nu_{x}+2 \nu_{y}=1+\Delta \nu$ with $\Delta \nu=0.01$.

\section{B. Difference resonance}

The effective Hamiltonian near the vicinity of the difference resonance: $\nu_{x}-2 \nu_{y}=p+\Delta \nu$, where $p$ is an integer, can also be derived similarly to the sum resonance and is given by,

$$
\begin{aligned}
\mathcal{H}= & \frac{2 \pi \Delta \nu}{5}\left(J_{x}-2 J_{y}\right) \\
& -\frac{\pi \Delta \nu C_{1002}}{\phi \sqrt{L} \sin \pi(p+\Delta \nu)} J_{x}^{1 / 2} J_{y} \cos \left(\psi_{x}-2 \psi_{y}\right) .
\end{aligned}
$$

Here $K=\left(\bar{x}^{2}+\bar{p}_{x}{ }^{2}\right)+\left(\bar{y}^{2}+\bar{p}_{y}^{2}\right) / 2$ is the invariance in the Hamiltonian system. Rewriting the Hamiltonian in the normalized coordinates, we have,

$$
\begin{aligned}
\mathcal{H}= & \frac{\pi \Delta \nu}{5}\left[\left(\bar{x}^{2}+\bar{p}_{x}{ }^{2}\right)-2\left(\bar{y}^{2}+\bar{p}_{y}{ }^{2}\right)\right] \\
& +\theta\left[\bar{x}\left(\bar{y}^{2}-\bar{p}_{y}{ }^{2}\right)+2 \bar{y} \bar{p}_{x} \bar{p}_{y}\right]
\end{aligned}
$$

where $\theta$ is given by,

$$
\theta=-\frac{\pi \Delta \nu C_{1002}}{2 \phi \sqrt{2 L} \sin \pi(p+\Delta \nu)}
$$




\section{Invariant tori}

Again, we would like to find a specific solution,

$$
\begin{aligned}
\bar{x} & =A_{x} \cos (2 \mu n), \quad \bar{p}_{x}=-A_{x} \sin (2 \mu n), \\
\bar{y} & =A_{y} \cos (\mu n), \quad \bar{p}_{y}=-A_{y} \sin (\mu n) .
\end{aligned}
$$

Note a different sign in the horizontal oscillation in comparison to the sum resonance. Solving the Hamilton's equation, along with the constant of motion, $K=A_{x}^{2}+A_{y}^{2} / 2$, we obtain,

$$
\begin{aligned}
& A_{x}=\frac{\pi \Delta \nu+\sqrt{\pi^{2} \Delta \nu^{2}+12 K \theta^{2}}}{6 \theta}, \\
& A_{y}=\frac{1}{3} \sqrt{\frac{-\pi^{2} \Delta \nu^{2}+12 K \theta^{2}-\pi \Delta \nu \sqrt{\pi^{2} \Delta \nu^{2}+12 K \theta^{2}}}{\theta^{2}}} .
\end{aligned}
$$

Unlike the sum resonance, the condition: $\pi^{2} \Delta \nu^{2}+$ $12 K \theta^{2} \geq 0$ is always satisfied and therefore does not provide any restriction on the stability.

Similarly, the special solution is compared against tracking as shown in Fig. 15. At small amplitude, we see good agreement between the theory and tracking. As the amplitude grows large, so does the deviation between the theory and tracking. It strongly reflects the higher order nonlinear effects.
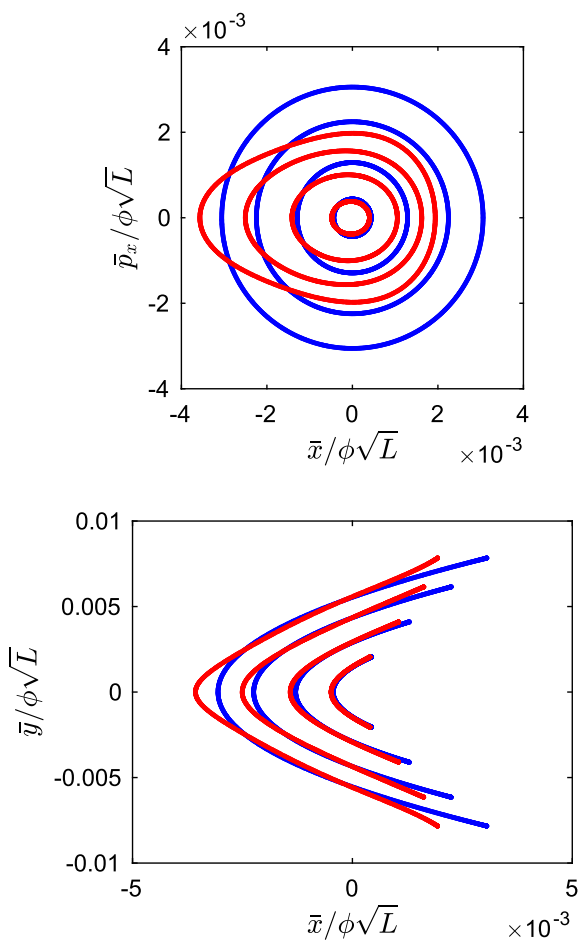

\section{Tune shifts}

It is well known [31] that the most important nonlinear effects in the next order are the amplitude-dependent tune shifts,

$$
\begin{aligned}
& \nu_{x}\left(J_{x}, J_{y}\right)=\nu_{x}+\alpha_{x x} J_{x}+\alpha_{x y} J_{y}, \\
& \nu_{y}\left(J_{x}, J_{y}\right)=\nu_{y}+\alpha_{x y} J_{x}+\alpha_{y y} J_{x},
\end{aligned}
$$

where the three coefficients can be computed using the normal form [17] or the Hamiltonian perturbation theory [26]. As outlined in Appendix C, here we have modified the normal form to avoid the so-called small denominator near the resonance. For the case being studied, we numerically evaluate the resonance normal form using differential algebra [32] and find $\alpha_{x x}=1.23 \times 10^{3} \mathrm{~m}^{-1}, \alpha_{x y}=-6.13 \times$ $10^{3} \mathrm{~m}^{-1}$ and $\alpha_{y y}=1.30 \times 10^{4} \mathrm{~m}^{-1}$. These values are quite large for a single cell, specially in the vertical plane.

Based on the normal form in Eq. (C8), the effective Hamiltonian in Eq. (31) is a function of the same nonlinear normalized coordinates as the fourth-order Hamiltonian that generates the tune shifts. Combining them according to the Cambell-Baker-Hausdorf $(\mathrm{CBH})$ theorem, we derive the fourth-order effective Hamiltonian,

$$
\begin{aligned}
\mathcal{H}= & \frac{\pi \Delta \nu}{5}\left[\left(\bar{x}^{2}+\bar{p}_{x}{ }^{2}\right)-2\left(\bar{y}^{2}+\bar{p}_{y}{ }^{2}\right)\right] \\
& +\theta\left[\bar{x}\left(\bar{y}^{2}-\bar{p}_{y}{ }^{2}\right)+2 \bar{y} \bar{p}_{x} \bar{p}_{y}\right]+\frac{\pi}{4}\left[\alpha_{x x}\left(\bar{x}^{2}+\bar{p}_{x}{ }^{2}\right)^{2}\right. \\
& \left.+2 \alpha_{x y}\left(\bar{x}^{2}+\bar{p}_{x}{ }^{2}\right)\left(\bar{y}^{2}+\bar{p}_{y}{ }^{2}\right)+\alpha_{y y}\left(\bar{y}^{2}+\bar{p}_{y}{ }^{2}\right)^{2}\right] .
\end{aligned}
$$
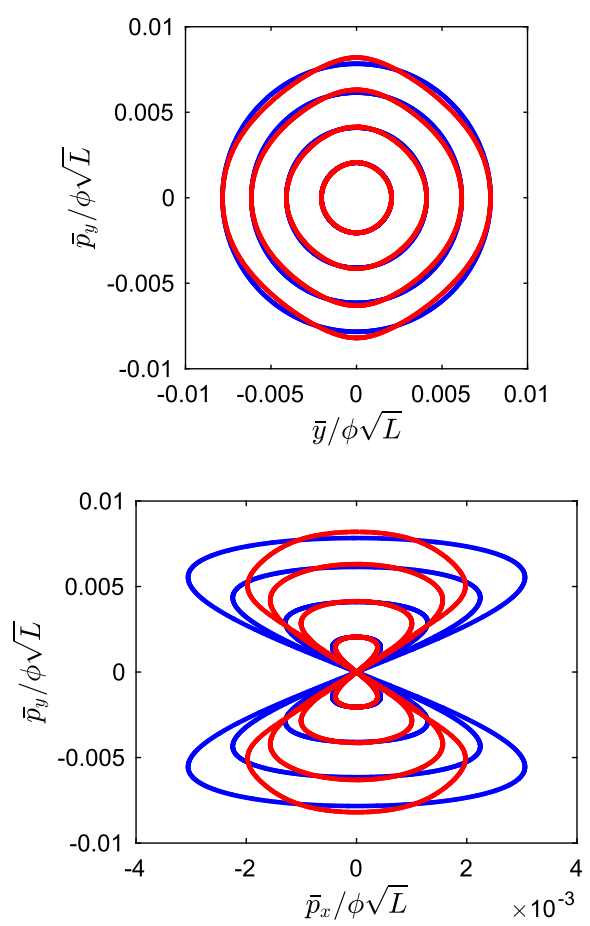

FIG. 15. A set of invariant tori in the normalized 4D phase spaces near the difference resonance: $\nu_{x}-2 \nu_{y}$ with $\nu_{x}=0.618$ and $\nu_{y}=0.314$. The red dots represent the orbits from tracking with bend length $L=5 \mathrm{~m}$ and angle $\phi=\pi / 64$ and the blue lines are the special solutions of the Hamiltonian in Eq. (31) with $\Delta \nu=-0.01$ and $\theta=72.81 \mathrm{~m}^{-1 / 2}$. 

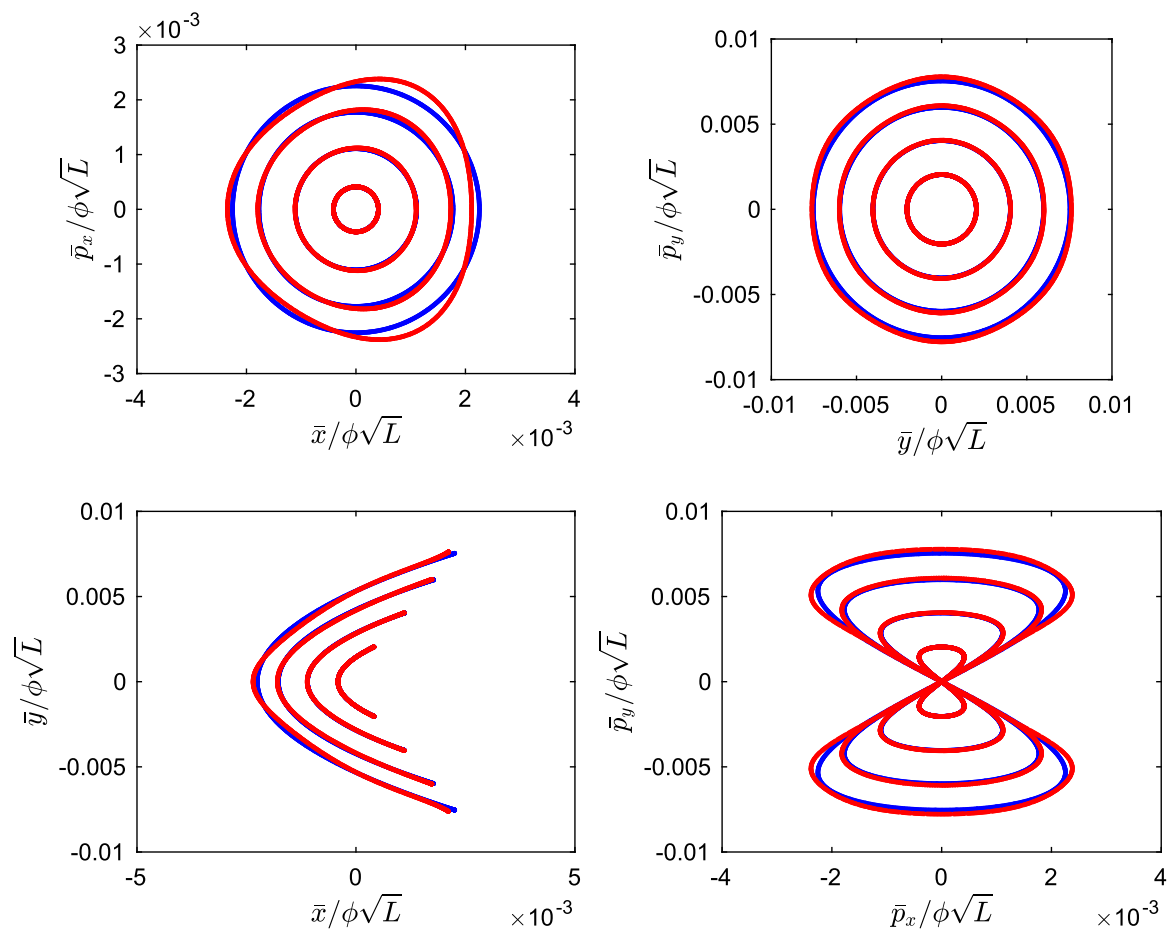

FIG. 16. A set of invariant tori in the nonlinear normalized 4D phase spaces near the difference resonance: $\nu_{x}-2 \nu_{y}$ with $\nu_{x}=0.618$ and $\nu_{y}=0.314$. The red dots represent the transformed periodic orbits from tracking and the blue lines are the special solutions of the Hamiltonian in Eq. (36).

The solution in Eq. (33) remains a special solution of the Hamilton's equation with the following conditions,

$$
\begin{aligned}
& \pi\left(\alpha_{x x}-4 \alpha_{x y}+4 \alpha_{y y}\right) A_{x}^{3}-6 \theta A_{x}^{2} \\
& \quad+2 \pi\left[\Delta \nu+\left(\alpha_{x y}-2 \alpha_{y y}\right) K\right] A_{x}+2 K \theta=0,
\end{aligned}
$$

and $A_{y}=\sqrt{2\left(K-A_{x}^{2}\right)}$. The cubic equation can be solved analytically using the third root: $x_{2}$ in Appendix A. The special solution is compared again to the tracking in the nonlinear normalized coordinates with the same $K$ values as shown in Fig. 16. The displayed orbits have been transformed to the normalized coordinates by a third-order Taylor map obtained in the normal form procedure as outlined in Appendix C.

\section{DOUBLE RESONANCES}

At the vicinity of two resonances: $\nu_{x}+2 \nu_{y}=p+\Delta \nu$ and $3 \nu_{x}=q+\delta \nu$, where $p, q$ are integers, the third-order effective Hamiltonian defined by the three-turn map can be derived similarly to the sum resonance. First, since the Lie operator associated with the integer $p$ and $q$ is again an identity, we have

$$
e^{:-3 \mathcal{H}_{0}:}=e^{:-\pi\left[2 \delta \nu J_{x}+(3 \Delta \nu-\delta \nu) J_{y}\right]:},
$$

where, $\mathcal{H}_{0}=2 \pi\left(\nu_{x} J_{x}+\nu_{y} J_{y}\right)$, is the free Hamiltonian. Secondly, we go through the same derivation in
Appendix B with the replacements, $-\frac{6 \pi \Delta \nu}{5} J \rightarrow-\pi\left[2 \delta \nu J_{x}+\right.$ $\left.(3 \Delta \nu-\delta \nu) J_{y}\right]$ and $\mu J \rightarrow \mathcal{H}_{0}$. As a result, Eq. (B6) should be replaced by,

$$
\begin{aligned}
-3 \mathcal{H}= & -\pi\left[2 \delta \nu J_{x}+(3 \Delta \nu-\delta \nu) J_{y}\right] \\
& +\frac{:-\pi\left[2 \delta \nu J_{x}+(3 \Delta \nu-\delta \nu) J_{y}\right]:}{\left(1-e^{: \mathcal{H}_{0}:}\right)} f_{3}^{(r)},
\end{aligned}
$$

where $f_{3}^{(r)}$ cab be read from Eq. (20),

$$
\begin{aligned}
f_{3}^{(r)}= & \frac{1}{\phi \sqrt{L}}\left[C_{3000} J_{x}^{3 / 2} \cos 3\left(\psi_{x}-\pi \nu_{x}\right)\right. \\
& \left.+C_{1020} J_{x}^{1 / 2} J_{y} \cos \left(\psi_{x}+2 \psi_{y}-\pi \nu_{x}-2 \pi \nu_{y}\right)\right] .
\end{aligned}
$$

Finally, after some lengthy but straightforward algebra, we obtain the effective Hamiltonian,

$$
\begin{aligned}
\mathcal{H}= & \frac{\pi}{3}\left[2 \delta \nu J_{x}+(3 \Delta \nu-\delta \nu) J_{y}\right] \\
& -\frac{\pi \delta \nu C_{3000}}{\phi \sqrt{L} \sin \pi(q+\delta \nu)} J_{x}^{3 / 2} \cos 3 \psi_{x} \\
& -\frac{\pi \Delta \nu C_{1020}}{\phi \sqrt{L} \sin \pi(p+\Delta \nu)} J_{x}^{1 / 2} J_{y} \cos \left(\psi_{x}+2 \psi_{y}\right) .
\end{aligned}
$$

Note that the resonance driving terms are identical to the ones in the case of the single resonance respectively. Again the small denominator problem is resolved since it does not become singular when $\delta \nu$ or $\Delta \nu$ approaches to zero. 

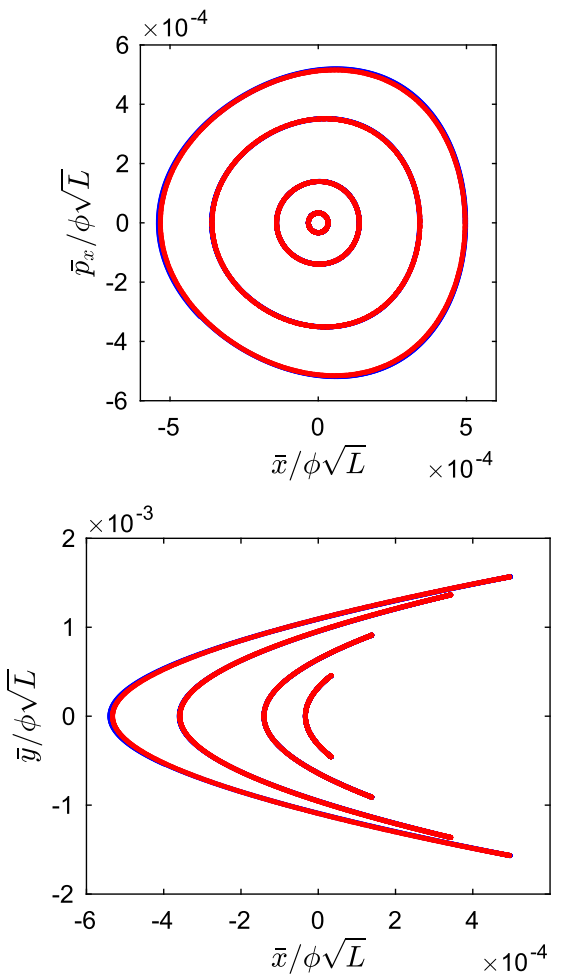
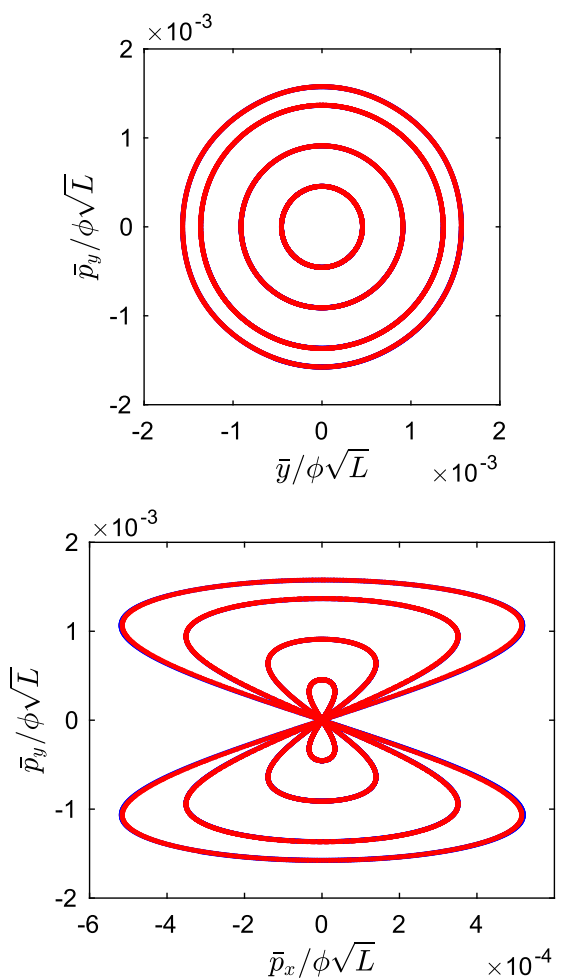

FIG. 17. A set of invariant tori in the nonlinear normalized 4D phase spaces on the resonance $3 \nu_{x}$ and near the sum resonance: $\nu_{x}+2 \nu_{y}$ with $\nu_{x}=2 / 3$ and $\nu_{y}=(1 / 6)+0.005$. The red dots represent the transformed periodic orbits from tracking and the blue lines are the numerical solution in Eq. (42).

Adding the tune shifts term, we write the fourth-order effective Hamiltonian in the normalized coordinates,

$$
\begin{aligned}
\mathcal{H}= & \frac{\pi}{6}\left[2 \delta \nu\left(\bar{x}^{2}+\bar{p}_{x}^{2}\right)+(3 \Delta \nu-\delta \nu)\left(\bar{y}^{2}+\bar{p}_{y}^{2}\right)\right] \\
& +\kappa \bar{x}\left(\bar{x}^{2}-3 \bar{p}_{x}^{2}\right)+\theta\left[\bar{x}\left(\bar{y}^{2}-\bar{p}_{y}^{2}\right)-2 \bar{y} \bar{p}_{x} \bar{p}_{y}\right] \\
& +\frac{\pi}{4}\left[\alpha_{x x}\left(\bar{x}^{2}+\bar{p}_{x}{ }^{2}\right)^{2}+2 \alpha_{x y}\left(\bar{x}^{2}+\bar{p}_{x}{ }^{2}\right)\right. \\
& \left.\times\left(\bar{y}^{2}+\bar{p}_{y}{ }^{2}\right)+\alpha_{y y}\left(\bar{y}^{2}+\bar{p}_{y}{ }^{2}\right)^{2}\right] .
\end{aligned}
$$

where,

$$
\kappa=-\frac{\pi \delta \nu C_{3000}}{2 \phi \sqrt{2 L} \sin \pi(q+\delta \nu)},
$$

and $\theta$ is given by Eq. (24).

The numerical solution of the Hamilton's equation is compared to the tracking in the nonlinear normalized coordinates with the same initial conditions as shown in Fig. 17. The displayed tracking orbits have been transformed to the normalized coordinates by a third-order Taylor map obtained in the normal form procedure. The parameters used in the numerical integration are tabulated in Table I. The agreement between the tracking and theory is excellent.

\section{CONCLUSION}

We have analytically solved the linear optics in a TME cell with three quadrupoles. The cell can be completely characterized by five independent parameters: its betatron tunes $\nu_{x}, \nu_{y}$, the dimensionless horizontal beta function at the center of the dipole $\bar{\beta}_{2}$, bending angle $\phi$, and length $L$ of the dipole. Ideally, $\bar{\beta}_{2}$ should be fixed according to a minimum emittance. Formulas of the lattice functions, emittance, and natural chromaticity are derived. Three sextupoles in two families are introduced to zero out the chromaticity.

After the chromatic compensation, we derive the complete third-order Lie factor, including chromatic and geometric aberrations. The chromatic aberration has been reduced to a minimum because of the local compensation and hence neglected. The geometric aberration contains an explicit overall factor of $1 / \phi \sqrt{L}$, leading to a scaling law, $\bar{A} \propto \phi \sqrt{L}$, of the dynamic aperture in the normalized phase

TABLE I. Parameters for the Double Resonances.

\begin{tabular}{lcccccc}
\hline \hline$\delta \nu$ & $\Delta \nu$ & $\theta\left[\mathrm{m}^{-1 / 2}\right]$ & $\kappa\left[\mathrm{m}^{-1 / 2}\right]$ & $\alpha_{x x}\left[\mathrm{~m}^{-1}\right]$ & $\alpha_{x y}\left[\mathrm{~m}^{-1}\right]$ & $\alpha_{y y}\left[\mathrm{~m}^{-1}\right]$ \\
\hline 0.00 & 0.01 & -90.2402 & -22.9766 & $-6.6074 \times 10^{2}$ & $1.3255 \times 10^{3}$ & $2.4174 \times 10^{4}$ \\
\hline \hline
\end{tabular}


space. We find that the dynamic aperture is much smaller than that in the FODO cell.

We have studied the third-order coupled resonances in the framework of the resonance normal form. Near a single sum resonance $\nu_{x}+2 \nu_{y}$, the third-order effective Hamiltonian is sufficient to explain the dynamics, including the dynamic aperture. It is a strong resonance resulting in a very small stable region, which is confined by the largest invariant tori.

For the single difference resonance $\nu_{x}-2 \nu_{y}$, again its invariant tori can be described by the effective Hamiltonian but with the tune-shift terms. More importantly, it is a weak resonance with a large stable region. The Hamiltonian seems not to define the stability. Finally, we find that the double resonances $3 \nu_{x}$ and $\nu_{x}+2 \nu_{y}$ can be analyzed similarly to the single resonance. Its stable region is also similar to the case of the single sum resonance $\nu_{x}+2 \nu_{y}$.

Our model is greatly simplified in comparison to realistic circular accelerators. Dynamics of the off-momentum particles can significantly differ from the on-momentum ones. Especially, we have ignored the high-order terms in energy for the chromaticity or momentum compaction factor, which can play an important role in full sixdimension tracking with synchrotron oscillation. To include these effects, the model has to be extended to include a straight section without any dispersion so that a rf cavity can be placed. A double-bend [33] or multibend [34] achromat could be a good choice for the next investigation.

\section{ACKNOWLEDGMENTS}

I would like to thank Yuri Nosochkov, Frank Schmidt, Robert Warnock, and Yiton Yan for helpful discussions. This work was supported by the Department of Energy under Contract No. DE-AC02-76SF00515.

\section{APPENDIX A: SOLUTION OF THE CUBIC EQUATION}

Setting $x=\sqrt{\bar{\beta}_{1}}$, the stability condition in Eq. (17) can be rewritten as a cubic equation,

$$
a x^{3}+b x^{2}+c x+d=0
$$

with the coefficients,

$$
\begin{aligned}
a= & -8 \sqrt{\bar{\beta}_{2}}\left(1+\cos 2 \pi \nu-\bar{\beta}_{2} \sin 2 \pi \nu\right), \\
b= & 3 \bar{\beta}_{2} \cos 3 \pi \nu+\bar{\beta}_{2}\left(47-2 \cos 2 \pi \nu_{y}\right) \cos \pi \nu \\
& +2\left[1-16 \bar{\beta}_{2}^{2}+\left(1-2 \bar{\beta}_{2}^{2}\right) \cos 2 \pi \nu+2 \bar{\beta}_{2}^{2} \cos 2 \pi \nu_{y}\right] \sin \pi \nu, \\
c= & 2 \sqrt{\bar{\beta}_{2}}\left[\left(4 \bar{\beta}_{2}^{2}-3\right) \sin 2 \pi \nu-8 \bar{\beta}_{2}(2+\cos 2 \pi \nu)\right], \\
d= & 8 \bar{\beta}_{2}\left(2 \bar{\beta}_{2} \cos \pi \nu+\sin \pi \nu\right) .
\end{aligned}
$$

The solution of the cubic equation is well known. One needs to compute first,

$$
\begin{aligned}
& \Delta_{0}=b^{2}-3 a c, \\
& \Delta_{1}=2 b^{2}-9 a b c+27 a^{2} d, \\
& \Omega=\frac{\Delta_{1}+\sqrt{\Delta_{1}^{2}-4 \Delta_{0}^{3}}}{2}
\end{aligned}
$$

And then the roots are given by,

$x_{k}=-\frac{1}{3 a}\left(b+\xi^{k} \Omega^{1 / 3}+\frac{\Delta_{0}}{\xi^{k} \Omega^{1 / 3}}\right), \quad k \in\{0,1,2\}$,

where $\xi=-\frac{1}{2}+\frac{i}{2} \sqrt{3}$. In this paper, we use $\bar{\beta}_{1}=x_{0}^{2}$.

\section{APPENDIX B: EFFECTIVE HAMILTONIAN OF THE SUM RESONANCE: $\nu_{x}+2 \nu_{y}$}

Near the sum resonance, it is well known in the canonical perturbation theory [35] that $J=J_{x}+2 J_{y}$ is the new action and $K=2 J_{x}-J_{y}$ is an invariance. Moreover, the free part of the Hamiltonian can be written as,

$-2 \pi \nu_{x} J_{x}-2 \pi \nu_{y} J_{y}=-\frac{2 \pi\left(\nu_{x}+2 \nu_{y}\right)}{5} J-\frac{2 \pi\left(2 \nu_{x}-\nu_{y}\right)}{5} K$.

Since $K$ is a constant of the motion, the second term can be dropped out from an effective Hamiltonian. To transfer to the rotating frame, we define the effective Hamiltonian by the three-turn map,

$$
\mathcal{M}^{3}=e^{:-3 \mathcal{H}} .
$$

This treatment is so obvious as in the case of $3 \nu_{x}$ [16], seeing a slower variation of the horizontal betatron phase every three-turn in the tracking. Here, we see a similar pattern in the tracking of the invariant tori, especially in the vertical plane.

Given $\mu=2 \pi\left(\nu_{x}+2 \nu_{y}\right) / 5$, we compute the three-turn map,

$$
\begin{aligned}
& \mathcal{M}^{3}=\left(e^{:-\mu J}: e^{: f_{3}^{(r)}}:\right)^{3}
\end{aligned}
$$

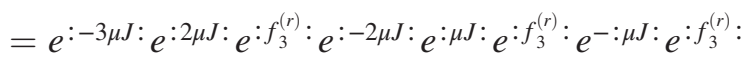

$$
\begin{aligned}
& =e^{:-3 \mu J:} e^{: e^{: 2 \mu J}: f_{3}^{(r)}}: e^{: e^{: \mu J:} f_{3}^{(r)}:} e^{: f_{3}^{(r)}:}
\end{aligned}
$$

We start with inserting two identity maps and then apply the similarity transformation to the Lie operators. Since the Lie operator associated with the integer $p$ is an identity, the first Lie operator is reduced to,

$$
e^{:-3 \mu J:}=e^{:-\frac{6 \pi \Delta \nu}{5} J}
$$

For the next three, we use the $\mathrm{CBH}$ theorem for the approximation up to the first order of $f_{3}^{(r)}$ and combine them into a single Lie operator, 


$$
\begin{aligned}
& e^{: e^{: 2 \mu J} f_{3}^{(r)}}: e^{: e^{: \mu J:} f_{3}^{(r)}:} e^{: f_{3}^{(r)}:} \\
& \approx e^{:\left(e^{: 2 \mu J}+e^{: \mu J:}+1\right) f_{3}^{(r)}:} \\
& =e^{:\left(\frac{1-e^{: 3 \mu J}:}{1-e^{; \mu J}:}\right) f_{3}^{(r)}:}
\end{aligned}
$$

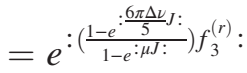

Applying the second form of the $\mathrm{CBH}$ theorem to combine two Lie operators in Eqs. (B4) and (B5) and using the definition of the effective Hamiltonian, $\mathcal{M}^{3}=e^{:-3 \mathcal{H}}$, we have,

$$
\begin{aligned}
-3 \mathcal{H} & \approx-\frac{6 \pi \Delta \nu}{5} J+\frac{:-\frac{6 \pi \Delta \nu}{5} J:}{\left(1-e^{: \frac{6 \pi \Delta \nu}{5} J}\right)} \frac{\left(1-e^{: \frac{6 \pi \Delta \nu}{5} J:}\right)}{\left(1-e^{: \mu J:}\right)} f_{3}^{(r)} \\
& =-\frac{6 \pi \Delta \nu}{5} J+\frac{:-\frac{6 \pi \Delta \nu}{5} J:}{\left(1-e^{: \mu J:}\right)} f_{3}^{(r)} .
\end{aligned}
$$

In addition, the resonance driving term can be read directly from Eq. (20),

$f_{3}^{(r)}=\frac{C_{1020}}{\phi \sqrt{L}} J_{x}^{1 / 2} J_{y} \cos \left(\psi_{x}+2 \psi_{y}-\pi \nu_{x}-2 \pi \nu_{y}\right)$.

After some straightforward algebra of computing the Poisson brackets, we obtain the Hamiltonian,

$$
\begin{aligned}
\mathcal{H}= & \frac{2 \pi \Delta \nu}{5}\left(J_{x}+2 J_{y}\right) \\
& -\frac{\pi \Delta \nu C_{1020}}{\phi \sqrt{L} \sin \pi(p+\Delta \nu)} J_{x}^{1 / 2} J_{y} \cos \left(\psi_{x}+2 \psi_{y}\right) .
\end{aligned}
$$

It is worth noting that it is consistent with the reflection symmetry and more importantly well behaved as $\Delta \nu$ approaches zero.

\section{APPENDIX C: NORMAL FORM IN VICINITY OF SINGLE RESONANCE OR DOUBLE RESONANCES}

We start from the well-known procedure of the normal form [17]. For a nonlinear Taylor map $\mathcal{M}$ truncated at an order $o$, we make a following transformation,

$$
\mathcal{R}^{-1} \mathcal{A} \mathcal{M} \mathcal{A}^{-1}=I_{2},
$$

where $\mathcal{A}^{-1}$ is a linear map that transforms to the normalized coordinates, $\mathcal{R}=e^{:-2 \pi\left(\nu_{x} J_{x}+\nu_{y} J_{y}\right)}$ the rotation maps, and $I_{2}$ is a nonlinear map near the identity. Its lowest perturbation is the second order, indicated with its subscript.

Similarly, we would like to make a transformation in the next order of perturbation,

$$
e^{-: N_{3}(z):} \mathcal{R}^{-1} e^{: F_{3}(z)}: \mathcal{A M A}^{-1} e^{-: F_{3}(z):}=I_{3} .
$$

Here we would like to absorb the second-order terms in $I_{2}$ to $F_{3}$ and $N_{3} . N_{3}$ is the driving term of a single resonance. Inserting an identity map after $e^{: F_{3} \text { : }}$

$$
e^{-: N_{3}(z):} \mathcal{R}^{-1} e^{: F_{3}(z):} \mathcal{R} \mathcal{R}^{-1} \mathcal{A M} \mathcal{A}^{-1} e^{-: F_{3}(z):}=I_{3} .
$$

and using Eq. (C1), and then performing a similarity transformation of the Lie operator $e^{: F_{3}:}$, we obtain,

$$
e^{-: N_{3}(z)}: e^{: F_{3}\left(\mathcal{R}^{-1} z\right):} I_{2} e^{-: F_{3}(z):}=I_{3} .
$$

To solve $F_{3}$ and $N_{3}$, we could rewrite this equation as,

$$
e^{-: N_{3}(z):} e^{: F_{3}\left(\mathcal{R}^{-1} z\right):} e^{: f_{3}^{(n)}(z)+f_{3}^{(r)}(z)}: e^{-: F_{3}(z):}=\bar{I}_{3},
$$

where $f_{3}^{(n)}+f_{3}^{(r)}$ is the Lie operator that generates $I_{2}$ and applying again the $\mathrm{CBH}$ theorem, we obtain

$$
e^{-: N_{3}(z)+F_{3}\left(\mathcal{R}^{-1} z\right)+f_{3}^{(n)}(z)+f_{3}^{(r)}(z)-F_{3}(z):}=\overline{\bar{I}}_{3} .
$$

The solution is given by,

$F_{3}(z)=f_{3}^{(n)}\left(\frac{1}{1-\mathcal{R}^{-1}} z\right), \quad N_{3}(z)=f_{3}^{(r)}(z)$.

It is clear from the solution that $N_{3}$ has been introduced to absorb all terms that have $1-\mathcal{R}^{-1}$ near zero value. Once $F_{3}$ and $N_{3}$ are calculated, we can compute $I_{3}$ using Eq. (C4) and proceed to the next order of perturbation.

This procedure can be continued until the right-hand side becomes identity due to the order of the truncation of the map. Inverting the Lie operators and maps, we derive the normal form presentation of the map,

$$
\begin{aligned}
\mathcal{M}= & \mathcal{A}^{-1} e^{-: F_{3}(\vec{z}):} \ldots e^{-: F_{o+1}(\vec{z}):} \mathcal{R} e^{: f_{3}^{(r)}(\vec{z}):} \ldots e^{: N_{o+1}(\vec{z}):} \\
& e^{: F_{o+1}(\vec{z})} \ldots e^{: F_{3}(\vec{z}):} \mathcal{A}
\end{aligned}
$$

[1] L. C. Teng, Minimizing the emittance in designing the lattice of an electron storage ring, Fermilab Report No. TM-1269, 1984.

[2] J. P. Potier and L. Rivkin, A low emittance lattice for the CLIC damping ring, in Proceedings of the Particle Accelerator Conference, Vancouver, BC, Canada, 1997 (IEEE, New York, 1997), p. 476.

[3] L. Rivkin, in Proceedings of the 7th International Workshop on Linear Colliders (LC97), Zvenigorod, Russia (INP, Provino, Russia, 1997), p. 644.

[4] P. Emma and T. Raubenheimer, Systematic approach to damping ring design, Phys. Rev. ST Accel. Beams 4, 021001 (2001).

[5] Y. Jiao, Y. Cai, and A. W. Chao, Modified theoretical minimum emittance lattice for an electron storage ring with extreme-low emittance, Phys. Rev. ST Accel. Beams 14, 054002 (2011). 
[6] F. Antoniou and Y. Papaphilippou, Analytical considerations for linear and nonlinear optimization of the theoretical minimum emittance cells: Application to the compact linear collider predamping rings, Phys. Rev. ST Accel. Beams 17, 064002 (2014).

[7] Y. Cai, Singularity and stability in a periodic system of particle accelerators, Phys. Rev. Accel. Beams 21, 054002 (2018).

[8] A. N. Kolmogorov, On conservation of conditionally periodic motions of small perturbation of Hamiltonian, Dokl. Akad. Nauk SSSR 98, 527 (1954).

[9] V. I. Arnold, Proof of a theorem of A. N. Kolmogokov on the invariance of quasiperoidic motions under small perturbations of the Hamiltonian, Usp. Mat. Nauk 18, 13 (1963) [Russ. Math. Surv. 18, 9 (1963)].

[10] J. Moser, On invariant curves of area-preserving mappings on an annulus, Nachr. Akad. Wiss. Gottingem, Math. Phys. K1 2, 1 (1962).

[11] A. Morbidelli and A. Giorgilli, Superexponential stability of KAM tori, J. Stat. Phys. 78, 1607 (1995).

[12] R. Hagedorn, Stability and amplitude ranges of two dimensional nonlinear oscillators with periodical Hamiltonian, Report No. CERN 57-1, 1957.

[13] A. Schoch, Theory of linear and nonlinear perturbations of betatron oscillations in alternating gradient synchrotrons, Report No. CERN 57-21, 1958.

[14] G. Guignard, A general treatment of resonances in accelerators, Report No. CERN 78-11, 1978.

[15] A. J. Dragt, Lie algebraic theory of geometrical optics and optical aberrations, J. Opt. Soc. Am. 72, 372 (1982); A. J. Draft, F. Neri, G. Rangarajan, D. R. Douglas, L. M. Healy, and R. D. Ryne, Lie algebraic treatment of linear and nonlinear beam dynamics, Annu. Rev. Nucl. Part. Sci. 38, 455 (1988).

[16] A. Chao, Lecture notes on topics in accelerator physics, Report No. SLAC-PUB-9574, 2002.

[17] E. Forest, M. Berz, and J. Irwin, Normal form methods for complicated periodic systems: A complete solution using differential algebra and Lie operators, Part. Accel. 24, 91 (1989).

[18] K. L. Brown, A first- and second-order matrix theory for the design of beam transport systems and charged particle spectrometers, SLAC Report No. 75; Adv. Particle Phys. 1, 71 (1968).

[19] D. A. Edwards and M. J. Syphers, An Introduction the Physics of High Energy Accelerators (John Wiley \& Sons, New York, 1993).
[20] E. D. Courant and H. S. Snyder, Theory of the alternatinggradient synchrotron, Ann. Phys. (N.Y.) 3, 1 (1958).

[21] Y. Cai, Symplectic maps and chromatic optics in particle accelerators, Nucl. Instrum. Methods Phys. Res., Sect. A 797, 172 (2015).

[22] Mathematica version 9, "A System for Doing Mathematics by Computer", Wolfram Research. Inc. (2014).

[23] H. Wiedemann, Radiation integrals, in Handbook of Accelerator Physics and Engineering, 2nd ed., edited by A. Chao, K. H. Mess, M. Tigner, and F. Zimmermann (World Scientific, Singapore, 2013), p. 220.

[24] H. Grote and F. C. Iselin, The MAD Program (Methodical Accelerator Design) Version 8.15, Report No. CERN/SL/ 90-13 (AP), 1990.

[25] A. J. Dragt and J. M. Finn, Normal form for mirror machine Hamiltonian, J. Math. Phys. (N.Y.) 20, 2649 (1979).

[26] R. D. Ruth, Single-particle dynamics in circular accelerator, AIP Conf. Proc. 153, 150 (1985).

[27] E. Levichev and V. Kvardakov, Nonlinear characteristics of the TME cell, RuPAC Proceedings, Novosibirsk, Russia, 2006, p. 327 (unpublished).

[28] D. Einfeld, Performance and perspective of modern synchrotron light sources, in Proceedings of eeFACT2016, Daresbury, UK, 2016, MOOTH4 (unpublished).

[29] Y. Cai, Single-particle dynamics in electron storage rings with extremely low emittance, Nucl. Instrum. Methods Phys. Res., Sect. A 645, 168 (2011).

[30] G. Franchetti and F. Schmidt, Extending the NonlinearBeam-Dynamics Concept of 1D Fixed Points to 2D Fixed Lines, Phys. Rev. Lett. 114, 234801 (2015).

[31] S. Y. Lee, K. Y. Ng, H. Liu, and H. C. Chao, Evolution of Beam Distribution in Crossing a Walkinshaw Resonance, Phys. Rev. Lett. 110, 094801 (2013).

[32] M. Berz, Differential algebra description of beam dynamics to very high order, Part. Accel. 24, 109 (1989).

[33] M. Sommer, Optimizing of the emittance of electrons (positrons) storage rings, Report No. LAL/RT/83-15, LAL, 1983.

[34] D. Einfeld, J. Schaper, and M. Plesko, A lattice design to reach the theoretical minimum emittance for a storage ring, in Proceeding of the 5th European Particle Accelerator Conference, Barcelona, Spain (Institute of Physics, Bristol, Philadelphia, 1996), p. 638.

[35] L. Michelotti, Phase space concept, AIP Conf. Proc. 184, 891 (1989). 